decreases the median survival to only 5 to 7 months with optimal therapy Thus, advances in treatment that may improve survival in these patients are highly desirable. METHODS: Tumor treating fields (TTFields) therapy (Novocure Ltd., Haifa, Israel) is an approved antimitotic treatment for patients with newly diagnosed and recurrent GBM. TTFields are low-intensity $(1-3 \mathrm{~V} / \mathrm{cm})$, intermediate-frequency $(100-300 \mathrm{kHz})$ alternating electric fields that selectively kill or arrest the growth of rapidly dividing cells by inhibiting the proper formation of the mitotic spindle and by causing rapid membrane breakdown during cytokinesis. RESULTS: Our center was the first in the world to apply TTFields treatment to histologically proven GBM in a pilot study of 20 individuals (10 GBM and 10 RGBM) in 2004 and 2005, and 4 of the original 20 patients are still alive today (2 GBM, 2 RGBM), in good health and no longer receiving any treatment roughly 15 years (range 14.215.9 years) after initiating TTFields therapy, with no clinical or radiological evidence of recurrence. The diagnosis of GBM was confirmed in all patients in two independent laboratories. Two of the 4 surviving patients exhibited radiological signs of tumor growth initially, before the tumor regressed in size after a median of 4 months of continuous treatment. CONCLUSIONS: Our results indicate that TTFields treatment may be remarkably successful for both newly diagnosed and recurrent GBM patients. We recommend that TTFields treatment should be applied for a sufficient amount of time, and that initial radiologic progression following treatment initiation should not be considered a reason to discontinue treatment.

\section{CTNI-71. TTFIELDS IN ROUTINE CLINICAL CARE OF NEWLY DIAGNOSED GBM PATIENTS IN GERMANY - FIRST REPORT ON THE FULLY ENROLLED TIGER STUDY POPULATION}

Oliver Bähr ${ }^{1}$, Ghazaleh Tabatabai ${ }^{2}$, Rainer Fietkau ${ }^{3}$,

Roland Goldbrunner ${ }^{4}$, and Martin Glas ${ }^{5} ;{ }^{1}$ Hospital AschaffenburgAlzenau, Aschaffenburg, Germany, ${ }^{2}$ University Hospital Tubingen, Tuebingen, Germany, ${ }^{3}$ University Hospital Erlangen, Erlangen, Germany, ${ }^{4}$ University Hospital Cologne, Cologne, Germany, ${ }^{5}$ Division of Clinical Neurooncology, Department of Neurology, University Hospital Essen, Essen, Germany

OBJECTIVE: Glioblastoma (GBM) is widely treated with surgery, chemoradiation and TTFields in combination with chemotherapy. Since the introduction of TTFields to first-line GBM treatment, prescribing physicians in Germany are interested to gain additional knowledge on the use in clinical practice. The TIGER study was designed to assess the therapy decision making process, safety and efficacy of TTFields in clinical routine, as well as evaluating quality of life (QoL) within 4 months after start of therapy, treatment compliance and duration. METHODS: This multi-center prospective non-interventional study in Germany (NCT03258021) included newly diagnosed GBM patients eligible for TTFields therapy. Within the study, after receiving introduction to TTFields therapy, patients could decide for or against receiving TTFields. The study was designed to recruit 500 patients for each group, with and without TTFields. Demographic data, QoL and reasons for therapy decision 2-4 months after treatment start are assessed using the EORTC-QLQ-C30/BN-20 and a TTFields questionnaire, respectively, with 18 months follow-up. RESULTS: At the last data cut-off (31 st March, 2020), the median follow-up was 11 months (range: 0-28 months) and $581(82 \%)$ of the 710 recruited patients in the trial had decided for TTFields therapy. According to preliminary analysis, this patient population represents a typical GBM population regarding median age (59 years, range: $19.0-85.0)$, gender (37\% female), median KPS (90, range: $40-100)$ and MGMT promoter methylation status (44\% methylated). In this population $49 \%$ received complete resection, $33 \%$ partial resection and $19 \%$ biopsy. CONCLUSION: The TIGER study allows prospective and systematic analysis of TTFields treatment decision making and use of typical GBM patient population in routine clinical care. In addition, the study allows the evaluation of compliance and treatment duration. Here, we will present an updated analysis of the TIGER study.

CTNI-72. PHASE 2 STUDY OF DIANHYDROGALACTITOL (VAL-083) IN PATIENTS WITH MGMT-UNMETHYLATED, BEVACIZUMABNAÏVE GLIOBLASTOMA IN THE RECURRENT AND ADJUVANT SETTING

Barbara O’Brien ${ }^{1}$, Marta Penas-Prado ${ }^{2}$, Carlos Kamiya-Matsuoka ${ }^{1}$, Shiao-Pei Weathers ${ }^{1}$, W K Alfred Yung ${ }^{1}$, Monica Loghin ${ }^{1}$, Rebecca Harrison ${ }^{1}$, Nazanin Majd ${ }^{1}$, Jeffrey Bacha ${ }^{3}$, Dennis Brown ${ }^{4}$, Greg Johnson ${ }^{5}$, John Langlands ${ }^{4}$, Richard Schwartz ${ }^{4}$, Sarath Kanekal ${ }^{4}$, Anne Steino ${ }^{4}$, Lorena Lopez ${ }^{4}$, and John de Groot ${ }^{1} ;{ }^{1}$ University of Texas MD Anderson, Houston, TX, USA, ${ }^{2} \mathrm{NIH} / \mathrm{NCI}$, Washington DC, USA, ${ }^{3}$ Delmar Pharmaceuticals Inc, Vancouver, BC, Canada, ${ }^{4}$ DelMar Pharmaceuticals Inc., Menlo Park, CA, USA, ${ }^{5}$ DelMar Pharmaceuticals, San Diego, CA, USA

Current standard-of-care for glioblastoma (GBM) includes surgery followed by concurrent therapy with radiation and temozolomide (TMZ) followed by adjuvant TMZ. Almost all GBM patients experience recurrent/ progressive disease despite upfront standard of care treatment, with a median survival after recurrence of 3-9 months. Unmethylated promoter for $\mathrm{O}^{6}$ methylguanine-DNA-methyltransferase (MGMT) is a validated biomarker for TMZ-resistance and is correlated with poor patient prognosis. VAL-083 is a bi-functional DNA-targeting agent which rapidly induces inter-strand cross-links at $\mathrm{N}^{7}$-guanine inducing double-strand breaks causing cell death and acts independent of MGMT DNA repair. This trial is an open-label two-arm biomarker-driven phase 2 clinical trial in MGMT-unmethylated bevacizumab-naïve GBM patients with either recurrent (Group 1) or newly diagnosed GBM requiring adjuvant therapy after chemo-radiation with temozolomide (Group 2). Patients receive VAL-083 IV at 30 or $40 \mathrm{mg} / \mathrm{m}^{2} / \mathrm{d}$ on days 1,2, and 3 of a 21-day cycle. The primary objective of this study is to determine the effect of VAL-083 on median overall survival (mOS) in MGMT-unmethylated recurrent GBM patients (Group 1); and progressionfree survival (PFS) in newly diagnosed GBM patients requiring adjuvant therapy after chemo-irradiation with temozolomide (Group 2), compared to historical controls in both groups. Tumor response will be assessed by MRI every 42 days, using RANO criteria. The initial starting dose in this study was $40 \mathrm{mg} / \mathrm{m}^{2} / \mathrm{d}$ on days 1,2 , and 3 of a 21 -day cycle, which was subsequently reduced to $30 \mathrm{mg} / \mathrm{m}^{2} / \mathrm{d}$ to improve tolerance due to myelosuppression. As of June 2-2020, 35 patients with recurrent GBM (Group 1) have received $40 \mathrm{mg} / \mathrm{m}^{2} / \mathrm{d}$ and 39 patients have received $30 \mathrm{mg} / \mathrm{m}^{2} / \mathrm{d}$ VAL- 083 . In the adjuvant setting (Group 2), 25 patients have been enrolled $\left(30 \mathrm{mg} / \mathrm{m}^{2} /\right.$ day) Enrollment, safety data and study updates will be presented at the meeting. Clinicaltrials.gov identifier: NCT02717962.

\section{CTNI-73. OVERALL SURVIVAL OF NEWLY DIAGNOSED GLIOBLASTOMA PATIENTS TREATED BY STANDARD THERAPY IN COMPARISON TO STANDARD THERAPY PLUS TUMOR TREATING FIELDS}

Aaron Rulseh, Adam Derner, Jan Sroubek, Jan Klener, and Josef Vymazal; Na Homolce Hospital, Prague, Czech Republic

BACKGROUND: Tumor treating fields (TTFields; $200 \mathrm{kHz}$ ) have shown significant prolonged survival in newly diagnosed (ndGBM). TTFields are anti-mitotic, low intensity, intermediate frequency alternating electric fields. The applied fields disrupt the mitotic spindle, microtubule assembly and the segregation of intracellular organelles during cell division, leading to apoptosis or mitotic arrest. We compared overall survival (OS) between patients recently treated with standard therapy and standard therapy plus TTFields at our institution. METHODS: Subjects $(\mathrm{N}=25)$ with ndGBM treated with standard therapy plus TTFields (STDTh-TTF) at our institution were included. Standard therapy (STDTh) consisted of surgical resection, followed by combined radiotherapy and chemotherapy (Temozolomide). In 3 cases, biopsies were performed instead of resection. The date of resection or biopsy was considered the entry date and was used in calculating survival. The study took place from July 2015 to April 2019. A matching control group of 25 subjects with ndGBM were treated with STDTh alone at our institution and were assembled from our database based primarily on date of resection or biopsy, and secondarily by age $(2$ subjects underwent biopsy in place of resection). When assembling the control group, the investigators were blinded to survival outcome. RESULTS: Significantly greater overall survival was observed for the group treated by TTFields in addition to standard therapy $(\mathrm{p}<0.001$; Hazard ratio [HR] $0.21 ; 95 \%$ confidence interval $[\mathrm{CI}]$ 0.1-0.45; median survival time STDTh-TTF 31.7 months, STDTh 7.1 months). The groups were balanced with respect to sex, and no differences with respect to age $(\mathrm{p}=0.13$; STDTh-TTF mean 51.58 years, SD 8.8 ; STDTh mean 52.42 years, SD 8.7$)$ or inclusion date $(\mathrm{p}=0.22)$ by paired t-test were detected. CONCLUSIONS: Our initial results appear promising with respect to overall survival benefit in patients undergoing TTFields treatment in addition to standard therapy.

CTNI-74. PHASE 3 TRIDENT TRIAL: CONCOMITANT RADIATION THERAPY (RT) AND TEMOZOLOMIDE +/- TUMOR TREATING FIELDS (TTFIELDS) IN NEWLY DIAGNOSED GLIOBLASTOMA

Wenyin Shi ${ }^{1}$, Lawrence Kleinberg ${ }^{2}$, Suriya A. Jeyapalan ${ }^{3}$, Samuel Goldlust ${ }^{4}$ Seema Nagpal ${ }^{5}$, Stephanie E Combs ${ }^{6}$, David Roberge ${ }^{7}$, Ryo Nishigawa $^{8}$, Rachel Grossman ${ }^{9}$, and Martin Glas ${ }^{10} ;{ }^{1}$ Jefferson University Hospital, Philadelphia, PA, USA, ${ }^{2}$ Johns Hopkins University School of Medicine, Baltimore, MD, USA, ${ }^{3}$ Tufts Medical Center, Boston, MA, USA, ${ }^{4}$ Hackensack University Medical Center, Hackensack, NJ, USA, ${ }^{5}$ Stanford University, Stanford, CA, USA, ${ }^{6}$ Technische Universität München (TUM), Munich, Germany, ${ }^{7}$ McGill University, Montreal, QC, Canada, ${ }^{8}$ Saitama Medical University, Saitama, Japan, ${ }^{9}$ Tel Aviv Medical Center, Tel Aviv, Israel, ${ }^{10}$ Division of Clinical Neurooncology, Department of Neurology, University Hospital Essen, Essen, Germany

BACKGROUND: Tumor treating fields (TTFields) is a non-invasive, regional antimitotic treatment approved as a standard-of-care for newly diagnosed glioblastoma (ndGBM). In the EF-14 Phase 3 trial, TTFields $(200 \mathrm{kHz})$ plus temozolomide (TMZ) significantly increased survival of 